\title{
Accuracy assessment of the monthly GRACE geoids based upon a simulation
}

\author{
E. J. O. Schrama - P. N. A. M. Visser
}

Received: 8 July 2005 / Accepted: 24 June 2006 / Published online: 29 August 2006

(C) Springer-Verlag 2006

\begin{abstract}
The purpose of this paper is to demonstrate the effect of geophysical background model errors that affects temporal gravity solutions provided by the Gravity Recovery And Climate Experiment (GRACE). Initial performance estimates by Dickey et al. (1997) suggested a formal geoid RMS error better than $0.1 \mathrm{~mm}$ up to spherical harmonic degree 5. Now that the GRACE gravity models and data are available, it is evident that these original expectations were too optimistic. Our hypothesis is that this is partially explained by errors in geophysical background models that need to be applied in the GRACE data reduction, and that this effect was not considered by Dickey et al. (1997). We discuss the results of a closed-loop simulation, where satellite trajectory prediction software is used for the generation of GRACE range-rate data and GRACE orbit solutions with the help of the Global Positioning System (GPS). During the recovery step in our closedloop simulation, we show that simulated nuisance signals (based on tide and air pressure model differences) map to a $0.7 \mathrm{~mm}$ geoid effect for periods longer than 3 months and to less than $0.4 \mathrm{~mm}$ for periods shorter than 3 months. The long-period geoid hydrology signal is at a level of $4.5 \mathrm{~mm}$, while the short-period hydrology is at $0.25 \mathrm{~mm}$. The long-period ocean bottom pressure (OBP) signal maps at $0.8 \mathrm{~mm}$ and for short periods it is $0.4 \mathrm{~mm}$. We conclude that short-period effects are difficult to observe by GRACE and that long-period
\end{abstract}

E. J. O. Schrama $(\varangle)$ ? P. N. A. M. Visser

Delft University of Technology,

Kluyverweg 1, 2629 HS Delft, The Netherlands

e-mail: e.j.o.schrama@tudelft.nl

P. N. A. M. Visser

e-mail: p.n.a.m.visser@tudelft.nl effects, like hydrology, are easier to recover than OBP variations.

Keywords Temporal gravity $\cdot$ Hydrology $\cdot$ Ocean bottom pressure - Tides · Air pressure - GRACE - GPS

\section{Introduction}

The Gravity Recovery And Climate Experiment (GRACE) mission, launched on 17 March 2002, consists of two satellites that fly in tandem, where inter-satellite distances are observed. The measurements are used to solve for separate potential coefficient sets on a monthly interval, where the eventual scientific interest goes to the recovery of mass variations at the surface of the Earth; see also Tapley et al. (2005). In Tapley et al. (2004b), it is demonstrated that the GRACE system saw a $10 \mathrm{~cm}$ annual variation in the equivalent water height over the Amazon river basin.

In order to reduce geoid errors and to focus on specific regional signals, Swenson and Wahr (2002) suggested a post-processing procedure that applies to the monthly geoid solutions currently provided by the Center of Space Research (CSR) at the University of Texas in Austin, USA, and the Geo-Forschungs Zentrum (GFZ) in Potsdam, Germany. The method discussed by Swenson and Wahr (2002) suggests a spatial averaging operator over a region of interest. Their method combines local information over a sufficiently large region in an attempt to reduce the GRACE geoid errors. Furthermore, the tendency in Tapley et al. (2004b) is to focus on annual signals in the resulting geoid maps, which is another way of suppressing the inherent noise in monthly geoid maps. 
The motivation for writing this paper is to quantify the GRACE monthly geoid errors that seem to partially appear as a North-South striping phenomenon in all solutions provided by CSR and GFZ. Our approach is an extension of the closed-loop simulation introduced by Visser and Schrama (2004), where the method now includes a post-processing residual analysis method that is described in this paper.

In Sect. 2, we start with a description of methods that were used in this paper. The closed-loop method enables us to simulate both geoid signals and errors and this enables us to quantify the capability of a GRACE system to retrieve a known signal in the presence of both measurement and background correction model noise; see also Velicogna et al. (2001) and Han et al. (2004).

The closed-loop simulation is a two-step procedure. In its first stage, a simulation data set is generated with the help of GEODYN orbit determination and parameter estimation software (Pavlis et al. 1999). GEODYN adjusts initial state vector differences of a computed orbit based upon simulated Global Positioning System (GPS) and GRACE low-low satellite-to-satellite (SST) tracking observations (referred to as the GRACE K/Kaband ranging system (KBR) observations).

New in this paper is a post-processing analysis method of GPS and KBR measurement residuals from which we recover monthly GRACE geoids. In Sect.3, we discuss the observability of hydrology and ocean bottom pressure (OBP), which are considered to be signals of scientific interest. Background model errors are simulated as ocean tide and atmosphere pressure differences. Conclusions of our closed-loop experiment and postprocessing method are discussed in Sect. 4.

\section{Method}

A primary observable of the GRACE system is the intersatellite range variation between two spacecraft separated by approximately $220 \mathrm{~km}$ and flying at $430 \mathrm{~km}$ altitude at the beginning of its mission. Both satellites carry a GPS receiver and we assume that there are separate methods, such as those described by Švehla (2005) and Švehla and Rothacher (2005), that allow one to obtain a trajectory with an accuracy of about $3 \mathrm{~cm}$ RMS relative to the International Terrestrial Reference Frame (ITRF). Furthermore, there are accelerometers on both spacecraft, which measure the skin acceleration on the spacecraft as a result of non-conservative forcing. This technique has been implemented on both Challenging Minisatellite Payload [CHAMP; launched on 15 July 2000 (Reigber et al. 2005b)] and GRACE (Tapley et al. 2004a).
The closed-loop experiment concept was introduced in Visser and Schrama (2004), where the orbits of both GRACE satellites are dynamically modeled with precision orbit determination (POD) software developed and maintained by the Space Geodesy Branch at the Goddard Space Flight Center in Greenbelt, MD, USA, (Pavlis et al. 1999). For the scope of this paper, we concentrate only on the modeling of conservative accelerations as a result of gravity, ocean tides, hydrology or OBP acting on the satellites, which is an assumption that avoids us from investigating the colored-noise characteristics of space-borne accelerometers.

Our hypothesis is that gravity field information should come from the differences between the GPS-derived positions of the spacecraft's centers of mass and a dynamical orbit computed with the help of POD software. The same is true for range-residuals between both GRACE satellites, which follow from the dynamical orbits and the observed inter-satellite ranges.

In Sect. 2.1, we repeat the main steps in the POD procedure as it is implemented in GEODYN; in Sects. 2.2 and 2.3, we discuss a residual post-processing analysis to yield improvements in either accelerations or the geopotential along the GRACE flight-path.

\subsection{Orbit prediction and parameter estimation}

Orbit prediction concerns all steps required to predict inertial positions and velocities of a satellite starting from an initial state vector and a priori dynamical models that are part of the equations of motion. In this context, parameter estimation is concerned with an estimation procedure to yield small improvements in either the initial state vector or components of the dynamical models that were used. There are several textbooks that describe both procedures (e.g. Seeber 1993), where it is explained that the motion of the satellite follows from:

$\ddot{\bar{x}}(t)=\nabla U(\bar{x}(t))+\sum_{i} \bar{f}_{i}(\bar{x}(t), t)$,

where $\bar{x}(t)$ is a position vector in an inertial reference frame at time $t$, while $U$ is the geopotential function and $\bar{f}_{i}$ are acceleration models for modeling atmospheric $\mathrm{drag}$, the gradient of the tide-generating potential, solid Earth tides, ocean tides, solar radiation pressure, relativistic corrections to the equations of motion, etc. Details of the numerical procedure to compute orbit are described in Seeber (1993) and Montenbruck and Gill (2000).

GEODYN is capable of predicting state vectors of both GRACE satellites and derived information ahead of time. A desired property is that changes in this predicted configuration can be related to variations in either 
the dynamical models [either in $U$ or in $\bar{f}_{i}$ in Eq. (1)] or the initial state vector (either $\bar{y}\left(t_{0}\right)$ or briefly $\bar{y}_{0}$ ).

There are several possibilities to implement such procedures. A generic method recommended by Press et al. (1989) is the shooting method for ordinary differential equations. This avoids one from implementing additional software and allows focus on the prediction problem. A drawback of this generic procedure is the efficiency of the method. For the sake of efficiency, most POD software, therefore, also contains separate differential equations, where small variations in the state vector at an epoch $t$ can be related to variations in either the initial state vector or variations in parameters contained within the dynamical models used.

The variational equations for an arbitrary dynamical parameter $\beta_{k}$ (index $k$ is used to identify one single dynamical parameter within a larger set) are:

$$
\frac{\partial \ddot{\bar{x}}(t)}{\partial \beta_{k}}=\frac{\partial \nabla U(\bar{x}(t))}{\partial \beta_{k}}+\sum_{i} \frac{\partial \bar{f}_{i}(\bar{x}(t), t)}{\partial \beta_{k}} .
$$

The conclusion is that a total of $6 \times K$ additional variational equations must be solved $\forall k \in[1, K]$. Modern POD software, like GEODYN, comes with all necessary options to compute variational equations for wellknown parameters within dynamical models and the variational equations that apply to the initial state vector problem. A special condition is the initial integration condition of Eq. (2), which depends on the type of $\beta_{k}$.

The application of Eq. (2) usually depends on the type of problem that is treated. In most cases, this system is evaluated for initial state vector variations, $C_{\mathrm{d}}$ parameters within an atmospheric density model, $C_{\mathrm{r}}$ parameters in solar radiation pressure models, and empirical dynamical accelerations in the across-track, along-track and radial directions, which model constant or onceper-revolution orbital dynamics that are inadequately represented with a priori dynamical models in Eq. (1).

After numerical integration of the variational equations, we get partial derivatives of the "orbit dynamics", which are not yet suited for observation types that we employ for POD. For this purpose, partial derivatives obtained from Eq. (2) are transformed into equivalent derivatives that apply to such observation types. (This step involves the generation of partial derivatives from the observations at a provided epoch to the relevant state vector partials that relate to the $\beta_{k}$ parameters defined for the problem.)

Once the observation equations are formed, a cost function is minimized in the least-squares sense. The least-squares estimation procedure considers all parameters that need to be estimated. Typically, these are the dynamical parameters $\beta_{k}$, as discussed above, but also station coordinate improvements, time bias estimates, tropospheric biases, etc. For details, see Pavlis et al. (1999).

The efficiency of the GEODYN POD method becomes one of the concerns for processing GRACE data. With $K$ dynamical parameters, the integrator must treat $6 \times(K+1)$ equations, whereas the evaluation of Eq. (1) contains the term $\nabla U$ that is normally computed with a spherical harmonic expansion up to degree and order $N_{\max }$; for details, see Heiskanen and Moritz (1967).

In Tapley et al. (2004a), we find $N_{\max }=160$, which means that the evaluation of Eqs. (1) and (2) requires operations of the order of $\alpha \times N \times\left(6+\beta+N_{\max }^{2}\right)$, where $\alpha$ depends on the implementation of the numerical integration procedure, $\beta$ depends on the number of modeled satellite-specific parameters, in which it is assumed that only the geopotential coefficients within $U$ are considered as dynamical parameters, and $N$ the number of steps at which the variational equations are computed. The conclusion is that the GEODYN POD procedure becomes computationally intensive when it is desired to form the full observation equations of a new gravity model.

To solve the resulting system of normal equations, a least-squares method minimizes the misfits between the observation data and the dynamical orbit solution, where we remark that standard Cholesky decomposition requires of the order of $P^{3}$ operations, where $P$ is the number of parameters involved. Another costly step is the assembly step of the normal equations; about $P^{2} \times N$ operations are required to compute the normal matrix, and roughly $P \times N$ operations are required for the right-hand side of the system of normal equations that follows from the least-squares method.

The GEODYN POD method was used by Visser and Schrama (2004) in a closed-loop simulation of 1 year of GRACE data. In principle, their simulated GRACE data set consists of 366 daily arcs for both GRACE satellites, where the GPS input is simulated through predicted inertial coordinates and the KBR input as an inter-satellite range observation. Adjusted in their simulation are initial state vectors of both GRACE satellites and normal equations are formed on a daily interval.

\subsection{GPS residual analysis}

In this section, we present a post-processing approach for the direct estimation of an empirical acceleration model of a satellite tracked by GPS. This method assumes that the orbit is determined in advance by means of kinematic or reduced dynamic orbit determination technique, and that the resulting state vectors are provided as in Švehla (2005), Švehla and Rothacher (2005) 
and van den IJssel and Visser (2003). The problem is now that the kinematic GPS satellite flight-path is not perfectly described by a priori dynamical models during orbit prediction.

In the following, we will derive the complete spectrum of perturbing accelerations by an alternative method that directly acts on the residuals obtained in the first step. If we label our "computed" orbit with subscript "c" then:

$\ddot{\bar{x}}_{\mathrm{c}}(t)=\nabla U\left(\bar{x}_{\mathrm{c}}(t)\right)+\sum_{i} \bar{f}_{i}\left(\bar{x}_{\mathrm{c}}(t), t\right)$,

and if there is an "observed" orbit, which is the GPSobserved path flown by the satellite that we label with subscript "o":

$$
\begin{aligned}
\ddot{\bar{x}}_{\mathrm{O}}(t)= & \nabla(U+T)\left(\bar{x}_{\mathrm{o}}(t)\right) \\
& +\sum_{i}\left(\bar{f}_{i}\left(\bar{x}_{\mathrm{o}}(t), t\right)+\Delta \bar{f}_{i}\left(\bar{x}_{\mathrm{o}}(t), t\right)\right)
\end{aligned}
$$

(with $T$ representing the disturbing geopotential) and if we assume that $\Delta \bar{x}(t)=\bar{x}_{\mathrm{O}}(t)-\bar{x}_{\mathrm{c}}(t)$ is observed by GPS, and that non-conservative accelerations $\bar{f}_{i}$ do not depend too heavily on displacements contained in $\Delta \bar{x}(t)$, then:

$$
\Delta \ddot{\bar{x}}(t)-\Gamma\left(\bar{x}_{\mathrm{c}}(t)\right) \Delta \bar{x}(t)=\nabla T\left(\bar{x}_{\mathrm{c}}(t)\right)+\sum_{i} \Delta \bar{f}_{i}\left(\bar{x}_{\mathrm{c}}(t), t\right),
$$

where $\Gamma()$ contains the second-order derivatives of the reference geopotential at $\bar{x}_{\mathrm{c}}(t)$. Equation (4) closely resembles the Hill equations as discussed in, e.g., Schrama (1992), and it offers a direct alternative for the computation of variational equations to estimate the missing accelerations in a dynamical model.

To estimate the missing accelerations $\bar{F}(t)$ along a computed orbit, we should evaluate:

$$
\Delta \ddot{\bar{x}}(t)-\Gamma\left(\bar{x}_{\mathrm{c}}(t)\right) \Delta \bar{x}(t)=\bar{F}(t) .
$$

The right-hand side of Eq. (6) contains the sum of all accelerations that were missing in the a priori dynamics used to predict the orbit. In the next section, we will introduce a delta model improvement (DMI) procedure, where we estimate the right-hand side of Eq. (5) from input contained in $\Delta \bar{x}(t)$.

\subsubsection{CHAMP GPS residual analysis}

To demonstrate the capabilities of the DMI procedure, we compute and validate an empirical acceleration model. We start with GPS kinematic and GPS reduced dynamic orbits for CHAMP, which were provided, respectively, by D. Švehla (personal communication) and J. van den IJssel (personal communication). Details of both procedures can be found in Švehla (2005),
Švehla and Rothacher (2005) and van den IJssel and Visser (2003).

Kinematic orbits as provided D. Švehla (personal communication) rely on the assumption that there is no dynamic behavior of the CHAMP satellite; CHAMP is treated as a roving receiver. Reduced dynamic orbits as provided by J. van den IJssel (personal communication) do assume "some" dynamics in the form of piecewise $900 \mathrm{~s}$ empirical accelerations. In both cases, the provided state vectors are corrected to the center of mass of CHAMP with the help of quaternion information provided by the CHAMP team at the GFZ.

Our goal is not to solve for a new gravity model from CHAMP GPS data; this is discussed by, e.g., Gerlach et al. (2003) and Ditmar et al. (2006). Instead, we want to quantify the accelerations required to correct our a priori model to fit the provided CHAMP orbits by a series of acceleration corrections derived from Eq. (6). In this case, our starting point is the GGM01S model developed at CSR (Tapley et al.2004a). For the remaining terms, we follow the IERS standards (McCarthy and Petit 2003) to formulate third-body gravitational effects, solid Earth tides, relativistic perturbations and an ocean tide model.

Our initial dynamical model does not contain any assumption with regard to the modeling of atmospheric drag or solar radiation pressure. Instead, we compute daily arcs where a constant and once-per-revolution acceleration model and an initial state vector correction are estimated. A generalized inverse technique based upon singular value decomposition is used to locate the minimum of the least-squares norm (Press et al. 1989).

Furthermore, the dynamic orbit is corrected by a time-series of acceleration corrections that is derived from Eq. (6). In the first iteration, we do not allow such corrections. In the second iteration, residual accelerations $\Delta \ddot{\bar{x}}(t)$ are computed with the help of a fast Fourier transformation (FFT) and a low-pass filter up to four cycles per orbital period (cpr), with a linear damping up to $8 \mathrm{cpr}$. (CHAMP state vectors were provided every $30 \mathrm{~s}$.

Equation (6) yields a direct estimate of the residual accelerations, and the obtained acceleration improvements are then used in the next iteration, where we fit our corrected dynamic orbit to the originally provided input orbits. This procedure is repeated twice. Radial orbit difference seen after iterations 2 and 3 are shown in Fig. 1. The length of the acceleration vector computed after the iterations 1 and 2 are shown in Fig. 2.

The conclusion from Figs. 1 and 2 is that the DMI procedure allows one to find purely dynamic orbit solutions that reproduce the GPS tracking data to be within $1-2 \mathrm{~cm}$ in all components. It should be remarked that along-track differences are systematically larger than 


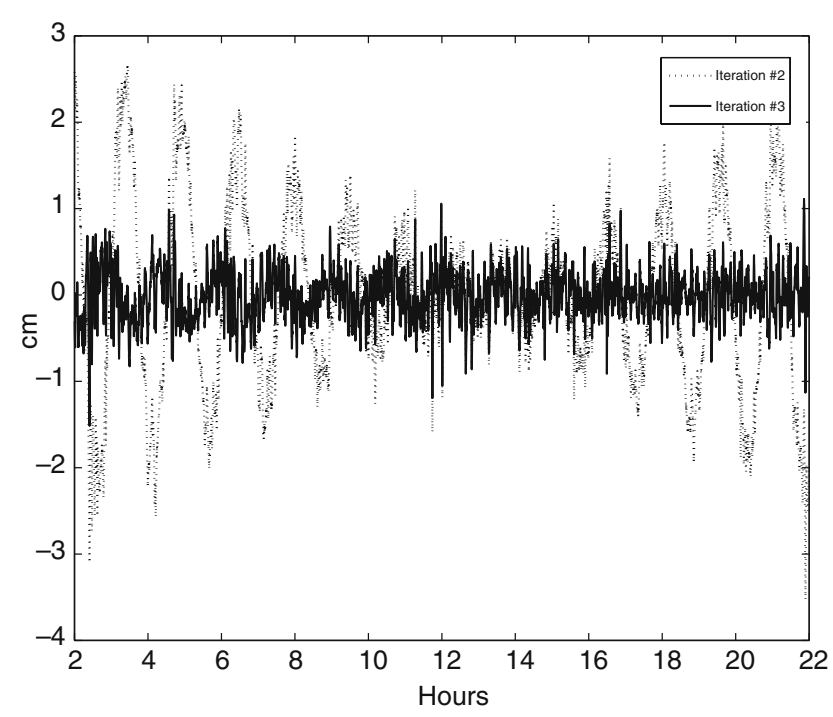

Fig. 1 Radial orbit differences during the DMI procedure. We show the radial perturbations after the second and third iterations, which are smaller than $5 \mathrm{~cm}$. Input to this procedure are the reduced dynamic state vector files provided by van den IJssel and Visser (2003) for CHAMP. The horizontal axis shows the time in hours relative to 1 January 2004 21:01:53 UTC. The vertical axis shows the perturbation effect (in $\mathrm{cm}$ ) of the computed orbit relative to the provided input state vector file

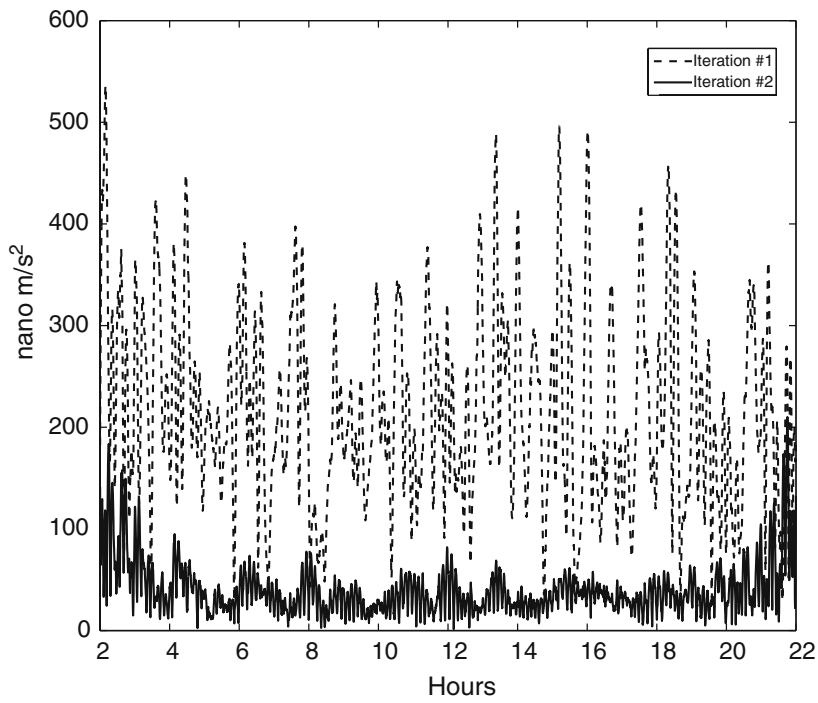

Fig. 2 The length of the acceleration vector by epoch estimated by the DMI procedure after the first and second iterations. Input are the differences between the provided input orbit and our dynamic orbits. The horizontal axis shows the time in hours relative to 1 January 2004 21:01:53 UTC. The vertical axis shows the acceleration effect (in $\mathrm{nm} / \mathrm{s}^{2}$ ) applied as a correction in the orbit determination software

radial or cross-track differences, which is expected from unmodeled accelerations affecting the orbit prediction problem; also see Seeber (1993) and Montenbruck and Gill (2000).
For the reduced dynamic orbit, we found for the result shown in Fig. 1 a cross/radial/along-track RMS of $0.0649 \mathrm{~m} / 0.4383 \mathrm{~m} / 4.4843 \mathrm{~m}$ before the DMI procedure was applied, and after the second iteration we obtained $0.0048 \mathrm{~m} / 0.0102 \mathrm{~m} / 0.0128 \mathrm{~m}$, where the first and last hours were omitted from the statistics due to edge effects. (Edge effects are characteristic for POD (Montenbruck and Gill 2000) and not necessarily related to the use of FFTs.) Similar results were obtained with the kinematic orbits provided by D. Švehla, although it should be mentioned that kinematic orbits are noisier, and that this is responsible for spikes in the residual acceleration spectrum.

We will not provide any further interpretation on the solved-for acceleration vector time-series, except to mention that the DMI procedure allows one to significantly improve orbit dynamics for satellites that are continuously tracked with GPS. The combination of constant and once-per-revolution accelerations and a spectrum of residual accelerations up to $4 \mathrm{cpr}$ with a linear damping up to $8 \mathrm{cpr}$ is, according to Fig. 1, sufficient for describing the CHAMP center of mass to be within the GPS-derived orbit accuracy, which is claimed to be at the $3 \mathrm{~cm}$ level for CHAMP (van den IJssel and Visser 2003).

In Fig. 2, the solved-for accelerations are usually less than $500 \mathrm{~nm} / \mathrm{s}^{2}$, which includes a variety of unmodeled effects that originate from radiation pressure and atmospheric drag-induced accelerations on CHAMP. In the second iteration of the DMI procedure, we find accelerations on the level of $10 \mathrm{~nm} / \mathrm{s}^{2}$ that correspond, according to Eq. (6), to a maximal displacement of $3.3 \mathrm{~mm}$.

A preliminary conclusion is that the obtained DMI spectra are probably hard to apply to temporal gravity research, essentially because the obtained accelerations are relatively noisy compared to the magnitude of the accelerations introduced by the hydrology signal. More details on this issue will be presented in Sect.2.2.2.

Our initial set-up was such that we only incorporate gravitational forcing (in this case the GGM01S model) and third-body accelerations and a solid Earth and ocean tide model; also see McCarthy and Petit (2003). To separate non-conservative and conservative accelerations from the obtained DMI spectra, one could either attempt to incorporate non-conservative models as in the GEODYN POD approach; see Pavlis et al. (1999). An alternative could be to employ the accelerometer of CHAMP, several examples are shown in Reigber et al. (2005a).

As far as efficiency is concerned, the DMI procedure is easier to implement than the GEODYN approach because the POD software only requires "forward" dynamics including a few variational equations 
for initial state vector and a generalized $1 \mathrm{cpr}$ and constant accelerations. (A day of CHAMP data as shown in the previous example requires no more than $120 \mathrm{~s}$ of computer time.) The main benefit is that the DMI procedure does not require any further assumptions on the parameterization of the missing dynamics.

A potential drawback is that the DMI procedure does not help in specifying the origin of the missing accelerations. Furthermore, it does not help to specify whether residual spacecraft GPS antenna eccentricities are to blame for a part of the solved-for acceleration signal. Finally, any other GPS-specific error such as a coordinate frame discrepancy between the frame used for the input orbit and the frame assumed in the orbit software is absorbed in the DMI acceleration spectrum.

\subsubsection{Hydrology signal in acceleration spectrum}

Figure 3 shows the hydrology signal represented as a radial acceleration at satellite altitude as seen in the first 30 days by GRACE- 1 in the simulation data set that was used by Visser and Schrama (2004). In this case, we show a map of the radial accelerations obtained by application of the DMI procedure discussed in Sect.2.2. To project the accelerations, we used utilities available from the generic mapping tools (GMT) (Wessel and Smith 1996); that is, minimum curvature smoothing and unweighted averaging in $1 \times 1$ degree cells.

The only purpose is to show that the accelerations introduced by the hydrology signal at satellite altitude are on the level of $10 \mathrm{~nm} / \mathrm{s}^{2}$, which is in our opinion very challenging compared to the noise level of the GRACE GPS orbits themselves, which are on the order of $30 \mathrm{~mm}$

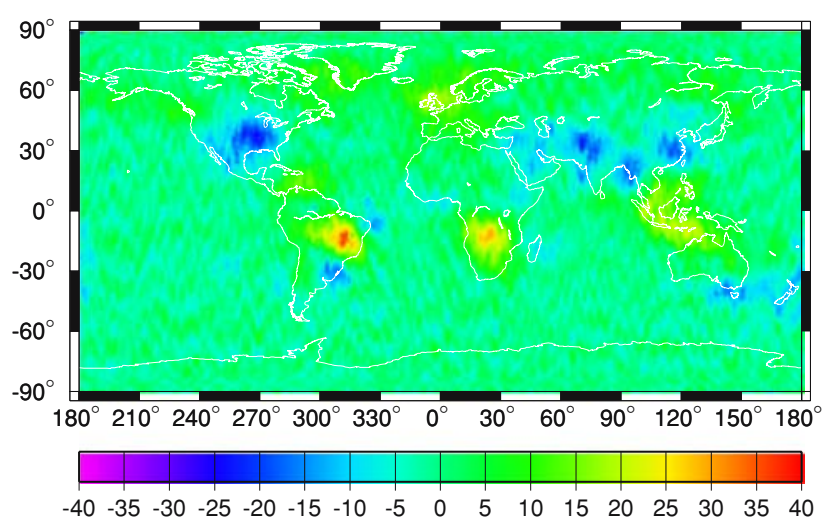

Fig. 3 Radial accelerations at satellite altitude as recovered from the first month in the GRACE simulation data set. The acceleration units are represented in $\mathrm{nm} / \mathrm{s}^{2}$, and they follow from the hydrology model used in the GRACE simulation
RMS. Effectively $10 \mathrm{~nm} / \mathrm{s}^{2}$ radially translates to a vertical displacement of $3.3 \mathrm{~mm}$, see also Eq. 6 where $\Gamma$ is multiplied by the $\Delta \bar{x}(t)$. The conclusion is that the GPS system in its current form is about a factor 10 , too noisy to allow it to see the hydrology signal as it was described by, e.g., Tapley et al. (2004a).

\subsection{Inter-satellite range residual analysis}

Results that we obtained from the closed-loop implementation that rely on GEODYN were explained in Visser and Schrama (2004), where initial state vector and geopotential coefficients up to degree and order 20 were solved for. Here, we assume that the KBR data becomes available as a velocity residual, and that this residual is the result of fitting an orbit solution to the observed GPS and KBR observation data.

In our case, we assume during the post-processing of the KBR residuals that the sum of the total residual energy is conserved for a satellite that is moving through a conservative force field, so that $v \Delta v=\Delta U$. If we follow the "KBR dot approach" in Jekeli (1999), Ray et al. (2003) and Rowlands et al. (2005) then:

$v_{0}\left(\Delta v_{2}-\Delta v_{1}\right)=\Delta U_{2}-\Delta U_{1}$

where $v_{0}$ is the reference velocity of the GRACE intersatellite baseline, $\Delta v_{1}$ and $\Delta v_{2}$ are variations in the velocity in the sense that they are observed by the KBR relative to computed values that follow from the POD software. The same is true for the variations in the potential $\Delta U_{1}$ and $\Delta U_{2}$ that hold for GRACE- 1 and GRACE2 ; these are values in the sense of "observed minus computed".

Our experience is that the differences in the potential must be corrected for additional model effects. An example of this effect is shown in Fig. 4, which follows from the right-hand side of Eq. (7). In Fig. 4, the potential difference is plotted as a function of time along 1 day in the simulated GRACE data set. In this case, the potential differences in this function do represent the effect of an unmodeled hydrology signal, i.e., the observed minus computed signal in the simulation setup refers to a situation where the "reference" GRACE orbits do not contain a hydrology effect, whereas the "truth" GRACE orbits do follow a hydrology effect.

A change in the potential on the right-hand side of Eq. (7) may also be the result of a geometric displacement effect that should be taken into account and that is unrelated to the presence of hydrology. This geometric correction was not mentioned by Jekeli (1999), Ray et al. (2003) or Rowlands et al. (2005), and we decided 


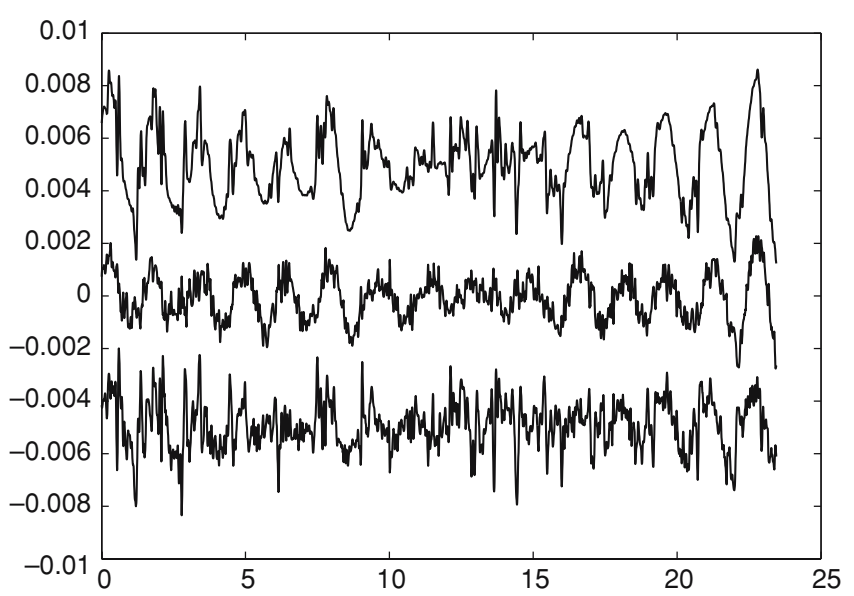

Fig. 4 Evolution of the right-hand side of Eq. (7) with and without the correction terms $\left[C_{1}, C_{2}\right.$ and $D$ in Eq. (8)]. The horizontal axis is in hours relative to 1 January 2004 in the GRACE hydrology simulation set. The upper line (displaced by $+0.005 \mathrm{~m}^{2} / \mathrm{s}^{2}$ ) is the left-hand side in Eq. (8), the center line is the geometric correction term $C_{1}-C_{2}$, and the lower line (displaced by $-0.005 \mathrm{~m}^{2} / \mathrm{s}^{2}$ ) is the evolution of the $\Delta U_{2}-\Delta U_{1}$ corrected for geometric effects

to implement it in Eq. (7) as:

$v_{0}\left(\Delta v_{2}-\Delta v_{1}\right)=\Delta U_{2}-\Delta U_{1}+C_{2}-C_{1}+D+e$

$C_{1}=\left(\bar{g}_{1}, \Delta \bar{y}_{1}\right)$

$C_{2}=\left(\bar{g}_{2}, \Delta \bar{y}_{2}\right)$

with $\bar{g}_{1}$ and $\bar{g}_{2}$ being the gravitational acceleration evaluated at GRACE- 1 and GRACE-2, and where $\Delta \bar{y}_{1}$ and $\Delta \bar{y}_{2}$ are orbit perturbations.

In a real-world situation, the presence of $C_{1}$ and $C_{2}$ is significant and effort must go into an accurate relative orbit determination of the KBR baseline vector. With $\sigma_{\mathrm{KBR}}=10^{-6} \mathrm{~m} / \mathrm{s} \mathrm{s}^{1 / 2}$ and $v_{0} \approx 7,500 \mathrm{~m} / \mathrm{s}$, we find from Eq. (8) $\sigma_{\Delta U}=10^{-2} \mathrm{~m}^{2} / \mathrm{s}^{2} \mathrm{~s}^{1 / 2}$, which is equivalent to a relative orbit error of about $1 \mathrm{~mm}$ as is claimed by Kroes et al. (2005).

Another term in Eq. (8) is:

$D=a_{1} \cos \left(\gamma_{1}\right)+a_{2} \cos \left(\gamma_{2}\right)$

where $\gamma_{1}$ and $\gamma_{2}$ denote small misalignment angles between the KBR baseline and both GRACE velocity vectors $\left(\gamma_{1}\right.$ and $\gamma_{2}$ are about $0.1^{\circ}$ and depend on argument of latitude). The variables $a_{1}$ and $a_{2}$ are estimated on a daily basis with a separate least-squares filter applied during a pre-processing step and this is outside the scope of the least-squares adjustment that takes care of determining potential coefficients on a monthly basis.

After the pre-processing step (a detrending method), we see that the residual orbit effect is reduced as is shown in Fig. (4). Our experience is that the detrending procedure as described above reduces the "trackiness" in the temporal geoid maps, which is a benefit of our procedure. So far the method discussed here results in a residual geopotential $\left(\Delta U_{2}-\Delta U_{1}\right)$ that is corrected for unwanted geometric effects.

After the detrending procedure, we obtain estimates for $\Delta U_{2}-\Delta U_{1}$ along the trajectories of both GRACE satellites, which is a measurement of the difference between the observed and the computed disturbance potential between GRACE-1 and GRACE-2. Our next goal is to obtain maps of the geoid which requires a least-squares procedure to invert this type of data. The observation equations are easily derived with the help of Heiskanen and Moritz (1967):

$$
\begin{aligned}
\Delta U_{2} & -\Delta U_{1}=\sum_{n=2}^{N_{\max }} \sum_{m=0}^{n} \sum_{a=0}^{1} \\
& \times g^{-1} \bar{C}_{\mathrm{nma}}\left(\frac{a_{e}}{r_{2}}\right)^{n+1} \bar{Y}_{\mathrm{nma}}\left(\phi_{2}, \lambda_{2}\right) \\
& -g^{-1} \bar{C}_{\mathrm{nma}}\left(\frac{a_{e}}{r_{1}}\right)^{n+1} \bar{Y}_{\mathrm{nma}}\left(\phi_{1}, \lambda_{1}\right),
\end{aligned}
$$

where $\bar{C}_{\text {nma }}$ are fully normalized potential coefficients, fully normalized spherical harmonics are indicated by $\bar{Y}_{\text {nma }}, g$ is the gravity acceleration at the Earth's surface with mean equatorial radius $a_{e}$ and $r_{1}, \phi_{1}, \lambda_{1}$ and $r_{2}, \phi_{2}, \lambda_{2}$ are the spherical polar coordinates of both GRACE satellites.

Within the closed-loop simulation and for runs that involve hydrology, air pressure or $O B P$ variations, we determined the potential coefficients of the disturbing potential $\bar{C}_{\text {nma }}$ in Eq. (12) to degree $N_{\max }=40$ with the help of a standard least-squares estimation procedure. We have selected $N_{\max }$ at twice the value used for the generation of the input fields which are based on a degree-20 spherical harmonic expansion. For our simulation involving ocean tides, we decided to raise $N_{\max }$ to 50 in Eq. (12), while the input fields were also generated at the same $N_{\max }$.

The preferred method for this problem is a straightforward set-up of the normal equations that follow. We did not invest time in setting up fast or efficient leastsquares methods since the amount of work can be handled within a day or two with a modern personal computer (PC) or workstation. Suitable a priori variance models are a latitude-weighted scheme, where the observation variance $\sigma_{\mathrm{o}}^{2}$ and parameter variance $\sigma_{\mathrm{p}}^{2}$ are chosen as:

$\sigma_{\mathrm{o}}^{2}=\frac{a_{2}}{a_{0} \cos \phi+a_{1}}$

$\sigma_{\mathrm{p}}^{2}=\frac{a_{3}}{n^{2}}$. 
Separate tests have shown that $a_{0}=1, a_{1}=10^{-3}$, $a_{2}=10^{-3}$ and $a_{3}=10^{-3}$ result in a tractable inversion scheme. The least-squares inversion is conducted over a period of 30 days, it involves $\approx 126,000$ observations and about 1,681 or 2,601 parameters depending on whether we take $N_{\max }=40$ or $N_{\max }=50$. The construction and inversion of the resulting normal matrices take about $72 \mathrm{~min}$ and $140 \mathrm{~min}$, respectively, on a modern PC for the discussed cases for monthly observation batches. Our post-processing method is systematically applied in Sect. 3 to compute changes in the geoid based on simulated geophysical signals in the GRACE closed-loop computation.

\section{Results}

In Sect. 2.1, we explained how existing POD techniques, like those implemented in the GEODYN software (Pavlis et al. 1999) can be used to process the GRACE data. For the closed-loop simulation results discussed here, two observation types were defined: one concerns the GPS orbit residuals of GRACE-1 and GRACE-2, and the second the inter-satellite range-rate residuals measured by the KBR on GRACE.

The simulated GRACE data set mentioned in Visser and Schrama (2004) has a length of 1 year, and consists of two satellites with a baseline orbit as for the GRACE configuration. For simplicity, we considered only conservative forces due to the Earth's gravity field, third-body accelerations and ocean tides. In total, we computed 366 daily arcs. The reference configuration starts on 1 January 2004 at a mean orbit altitude of $429 \mathrm{~km}$ relative to a sphere with a radius $6,378,137 \mathrm{~m}$.

During the data reduction runs, GEODYN is used to adjust the initial state vector. This program solves the variational problem that follows from Eq. (2) for the above-discussed configuration of satellites and observables. The low-low SST observations are $30 \mathrm{~s}$ integrated Doppler with an observation sigma of $1 \mu \mathrm{ms}^{1 / 2}$. The space-borne GPS observations are simulated at $2 \mathrm{~min}$ intervals as a coordinate observation type with an a priori sigma of $1 \mathrm{~cm}$. (The value of $1 \mathrm{~cm}$ is required to stabilize the normal equations, which become singular when low-low SST-only observations are used.)

The simulated observations themselves are assumed to be free of observation noise, and the abovementioned sigma's are only relevant for weighing their relative variance in the least-squares adjustment. (This procedure is in essence a regularization method). For the closedloop simulation, a "computed" configuration is identical to a case where a reference model is used during orbit prediction while an "observed" configuration is identical to the application of a "truth" model. Here, the GEODYN data reduction runs attempt to minimize observation residuals by tuning initial state vectors. This procedure results in daily files where the output consists of: (1) observed minus computed orbit residuals for both GRACE satellites and (2) and observed minus computed values for the velocity residual $\Delta v$ along the KBR baseline.

New in this paper is that the geoid maps are computed from the simulated orbit residuals and KBR velocity residuals, which are input to a post-processing technique that is based upon the theory explained in Sect. 2.3. With the help of a least-squares estimator, we then compute a best-fitting potential $\Delta U$ that is downward-continued to a sphere with radius $a_{e}$. A separate issue during the computation of the monthly geoid maps is that we incorporate a $5^{\circ}$ block averaging parameter to further reduce striping effects. This spatial filter is separate from the least-squares estimator, and it applies when the spherical harmonic coefficients are converted into $1 \times 1$ degree geoid grids.

In total, we acquire 12 monthly maps that, in turn, are input to a separate signal analysis procedure where each grid node is processed separately into a short- and a long-periodic part. Based upon the length of the simulation data set and the fact that monthly geoids were computed, we define the threshold to be 3 months. The rationale is that any temporal geoid signal at a period shorter than 3 months resembles the short-periodic fluctuations of the geoid signal; also see Thompson et al. (2004). The long-periodic fluctuations indicates how geoid signals map to periods greater or equal to 3 months.

The above-described procedure is applied to four separate simulations discussed in the following sub-sections.

\subsection{Hydrology}

In Fig. 5, we show the recovered long-period geoid RMS as a result of continental model hydrology. In this case, the truth model is based upon the hydrology model developed by Fan and van den Dool (2005) (relative to the year 2000), while the reference data set does not contain a hydrologic signal. The residual geoid effects are modeled up to $N_{\max }=40$, which is twice the value used for the input hydrology data set that was developed in spherical harmonics up to $N_{\max }=20$.

The conclusion from this calculation is that we are able to detect the main structures from the continental hydrologic signal by comparing it to the used model hydrology (not shown here). We conclude that all known dipoles are present in the recovered long periodic geoid RMS. The remaining signal RMS that maps to shorter periods is smaller than $0.5 \mathrm{~mm}$ RMS and it is shown in 


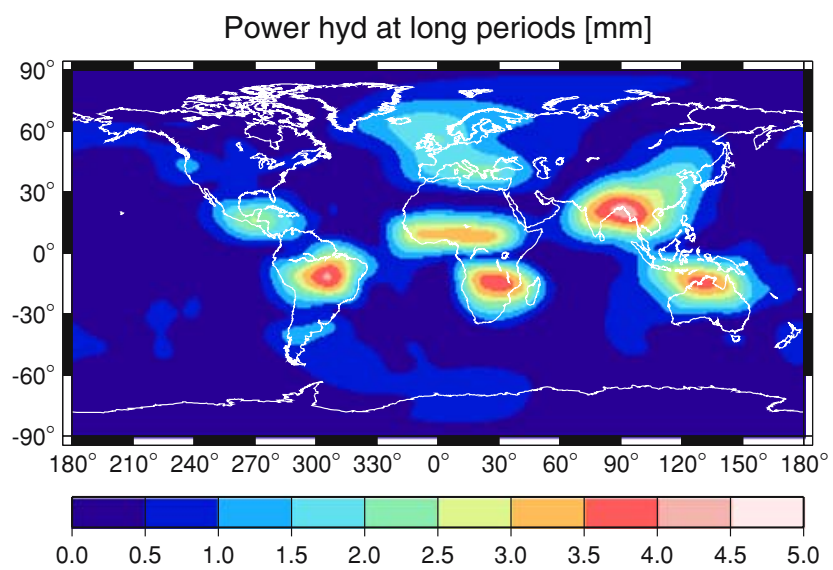

Fig. 5 Recovered long-periodic geoid RMS based upon the input hydrology from Fan and van den Dool (2005); scale mm

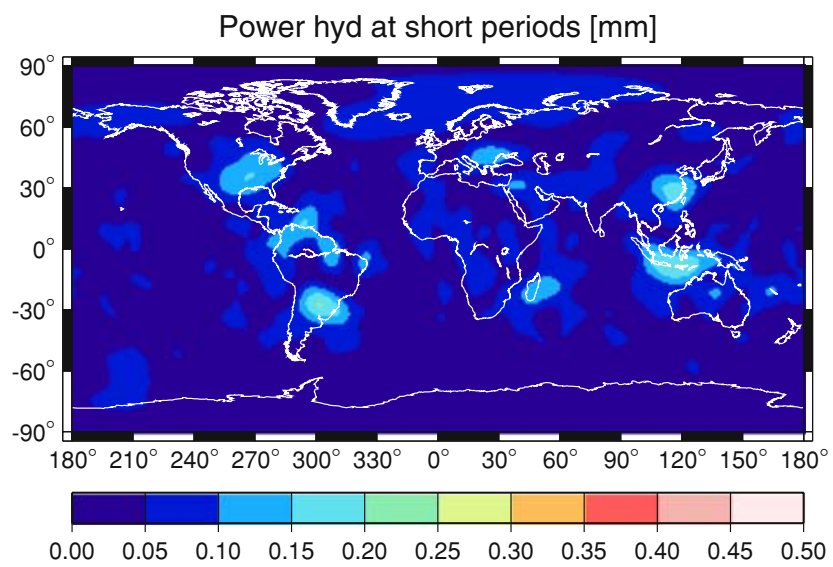

Fig. 6 Recovered short-periodic geoid RMS based upon the input hydrology from Fan and van den Dool (2005); scale mm

Fig. 6. Here, we conclude that the short periodic hydrology signal is substantially smaller.

Our current results are mostly free of striping effects, which is only possible with a suitable choice of the a priori observation and parameter variance $\sigma_{\mathrm{o}}^{2}$ and $\sigma_{\mathrm{p}}^{2}$ as in Eqs. (13) and (14) and the use of an exact solver for the normal equations. The natural sensitivity of the GRACE system is in the North-South direction. Our experience is that over-weighting $\sigma_{\mathrm{o}}$ relative to $\sigma_{\mathrm{p}}$ results in the striping pattern characteristic for the monthly GRACE geoid maps. Another cause for stripes in monthly geoids is the use of a block-diagonal solver that we initially used in which case the correlations between different orders $m$ are ignored in the problem.

\subsection{Air pressure}

Our post-processing method applied to the closed-loop simulation data set also offers the possibility to assess the consequences of error structures typical for geophysical background models. The rationale is that the GRACE data processing groups apply such background models to remove known gravitational effects caused by, for instance, air pressure and ocean tide variations (Tapley et al. 2004b).

For the air pressure part, we simulated this type of error as the difference between the European Center for Medium-Range Weather Forecasts (ECMWF) surface pressure reanalysis model and the National Centers for Environmental Prediction (NCEP) reanalysis surface pressure model. The former is provided with a spatial resolution of $0.5^{\circ}$ on 6 hourly grids, whereas the latter is provided on daily grids with $2.5^{\circ}$ spatial resolution. The assumption is that meteorological errors are represented by differences between two well-known models. In the closed-loop simulation, this means that the ECMWF model is used as a reference model while the NCEP reanalysis model is used as the truth.

The RMS of the recovered long-periodic geoid difference is shown in Fig. 7, while the short-periodic RMS is shown in Fig. 8. The conclusion from this computation is that the simulated surface air pressure errors do translate into a geoid RMS of $0.5 \mathrm{~mm}$ at latitudes less than $70^{\circ} \mathrm{N}$ or $70^{\circ} \mathrm{S}$, while larger variations (1.5 mm RMS) are observed over the poles.

The short-periodic air pressure difference geoid RMS is shown in Fig. 8. An unrealisticly large feature appears over the South Pole and a smaller one over Greenland. Apparently the ECMWF and the NCEP models differ in these regions, which is probably due to the shortage of polar meteorological observations and the data assimilation strategy followed by the different analysis centers. A possible remedy is to gather additional information,

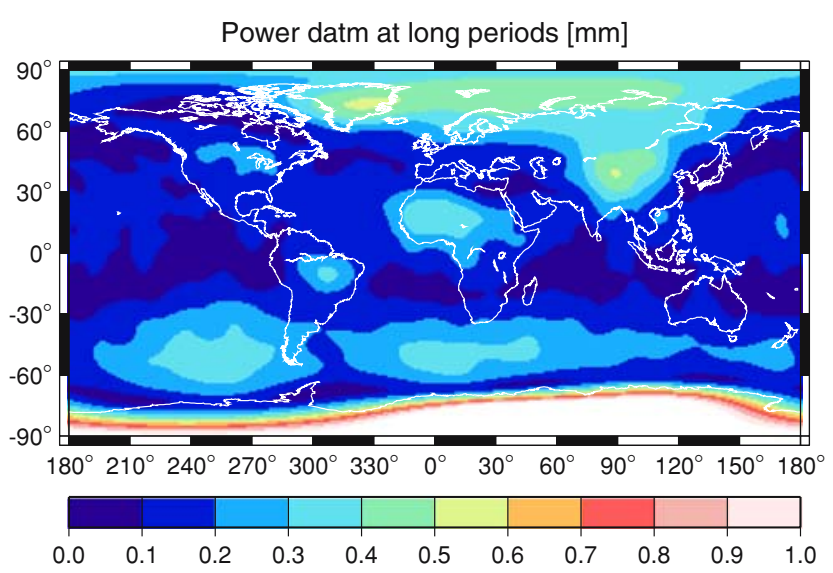

Fig. 7 Recovered long-periodic geoid RMS (in $\mathrm{mm}$ ) based upon difference between the NCEP reanalysis model and the ECMWF air pressure model 


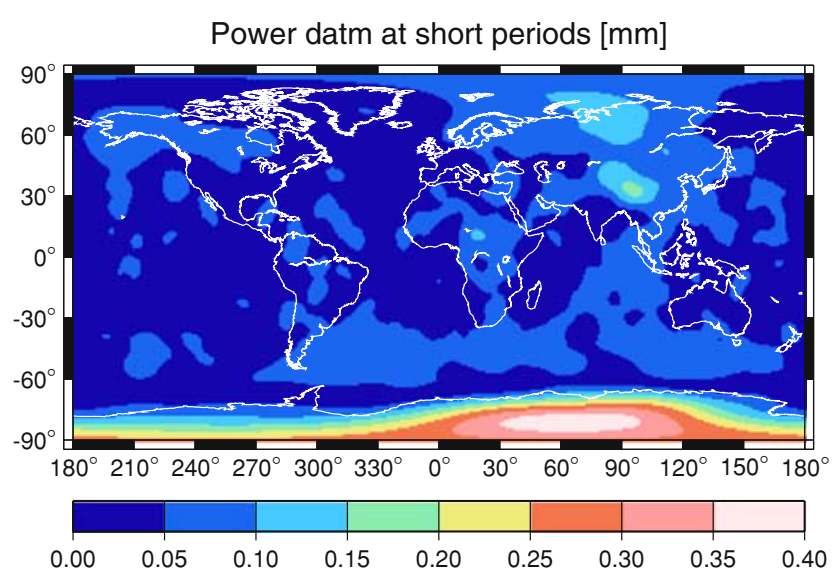

Fig. 8 Recovered short-periodic geoid RMS (in $\mathrm{mm}$ ) based upon difference between the NCEP reanalysis model and the ECMWF model

for instance from GPS occultation profiles that yield unique information about the vertical structure of the refractive index, which offers the possibility to estimate air pressure variations in the analyzed column.

\subsection{Ocean tides}

It was suggested by Schrama (2003) that ocean tide models may contain errors that affect geoid maps provided by the GRACE system. Ocean tides are nowadays well mapped by TOPEX/Poseidon (T/P) and Jason-1 satellite altimetry. The accuracy of tides in the deep ocean is usually better than $3 \mathrm{~cm}$ when all constituents are considered; for constituent $M_{2}$ we find an RMS of about $1.5 \mathrm{~cm}$, while other significant constituents such as $K_{1}$, $S_{2}$ and $O_{1}$ come with RMSs smaller than $1.0 \mathrm{~cm}$ in the deep ocean areas. The mentioned RMS of fit of ocean tide models is only warranted for latitudes within the inclination range of the $\mathrm{T} / \mathrm{P}$ altimeter system.

Tidal model errors increase in shallow seas because the spatial structure of the tidal wave shortens while the inter-track spacing of the satellite altimeter remains the same. Also, the quality of satellite altimetry data is affected by various factors in coastal and polar regions such as spurious radar backscatter from land and ice, and our inability to improve the altimeter sea-state bias beyond a certain level. Furthermore, tide model errors increase in polar regions because of the quality of polar hydrodynamic models that rely on few in situ observations, i.e., there is no $\mathrm{T} / \mathrm{P}$ altimeter data (and ERS-1/2 data is less accurate for observing ocean tides). Compared to the open ocean, a tide model in polar regions becomes more and more dependent on adequate dynamical models that must include realistic spatial distributions of tidal drag. Alternatively, we are faced with

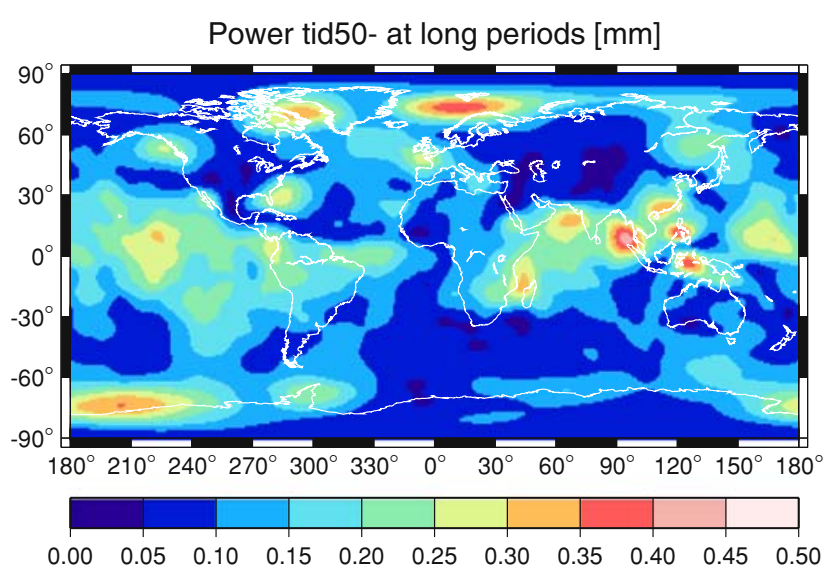

Fig. 9 Recovered long-period geoid RMS (in $\mathrm{mm}$ ) based upon the difference between the FES99 and the GOT99.2 ocean tide models

dedicated tide models that handle motions under ice shelves.

For the closed-loop calculations, we investigate the differences between the FES99 model (Lefèvre et al. 2002) and the GOT99.2 model (Ray 1999). The main reason for selecting these models is that they are documented and that they rely on different processing strategies. We selected the GOT99.2 ocean tide model in the reference run, while the FES99 finite element model is used as the truth in a GEODYN data reduction run.

One result from this simulation is the long-periodic map of geoid differences due to ocean tide model errors whose RMS is shown in Fig. 9, while the short-periodic equivalent is shown in Fig. 10. From these computations, we conclude that the long-periodic geoid RMS is approximately $0.5 \mathrm{~mm}$, and that the short-periodic geoid RMS is smaller than $0.2 \mathrm{~mm}$, which makes it one of the significant contributions to background tide model errors.

The natural question arises whether it would be possible to estimate the ocean tide from the GRACE data. One important issue would be the sampling of the tide signal by the GRACE tandem mission, which was discussed in Ray et al. (2003). The main conclusion is that GRACE was never optimized for being able to observe this type of signal as it was done for the T/P mission for tides. Nevertheless, there are advances in this area, such as in Han et al. (2005), who have claimed to be able to see an unmodeled ice shelf ocean tide effect in the GRACE data.

\subsection{Ocean bottom pressure}

The last effect that we consider in this paper is the OBP variation as predicted by the Estimating the Circulation 


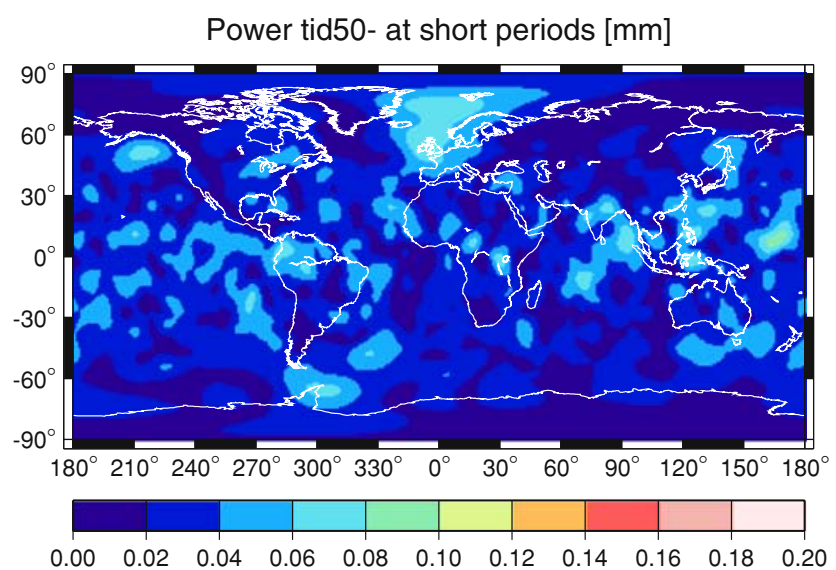

Fig. 10 Recovered short-period geoid RMS (in $\mathrm{mm}$ ) based upon the difference between the FES99 and the GOT99.2 ocean tide models

and Climate of the Ocean (ECCO) model. The ECCO model is maintained by a consortium of the Jet Propulsion Laboratory (JPL), Massachusetts Institute of Technology (MIT) and Scripps Institution of Oceanography (SIO); see Stammer et al. (1999). We have used the OBP predicted by the ECCO model, which is mainly the result of wind and atmospheric pressure forcing on the model. In the simulation, the reference run does not contain the effect, and in the GEODYN data reduction run, the ECCO model is used as the truth.

Figures 11 and 12 display how the simulated OBP signal maps into long- and short-period geoid RMS. Both maps appear to reflect extremes in the inverse barometer (IB) response (Mathers and Woodworth 2001), which occur at $100^{\circ} \mathrm{E} / 55^{\circ} \mathrm{S}$ and $90^{\circ} \mathrm{W} / 55^{\circ} \mathrm{S}$. Furthermore, there are several local excursions in coastal zones that seem to be reasonable for a wind-driven ocean model.

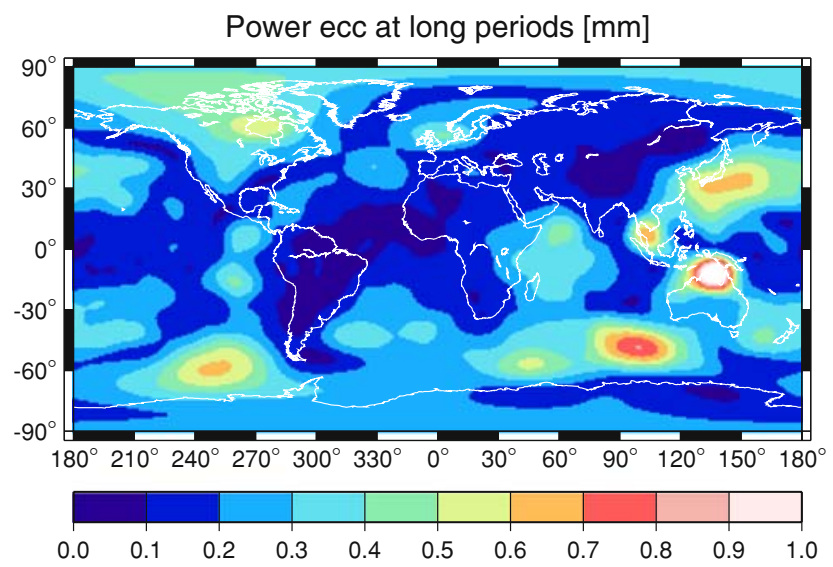

Fig. 11 Recovered long-period geoid RMS (in $\mathrm{mm}$ ) based upon the ECCO model ocean bottom pressure signal

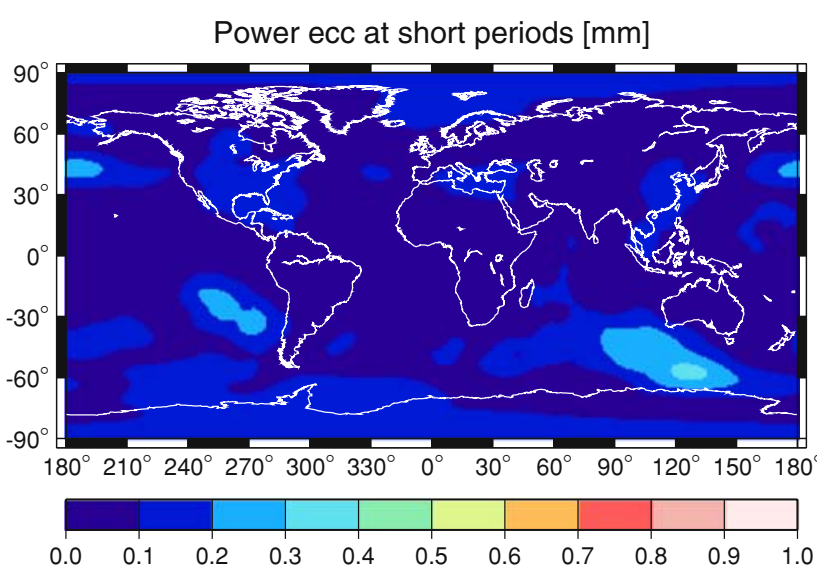

Fig. 12 Recovered short-period geoid RMS (in $\mathrm{mm}$ ) based upon the ECCO model ocean bottom pressure signal

Altogether, it must be concluded that the OBP signal is about a factor 4 smaller than the hydrologic signal, and that it is much harder to retrieve from the GRACE data because of the presence of tide and atmospheric pressure errors. The long-periodic features of the OBP signal stand out, and this can help its recovery from the GRACE data.

\section{Conclusions and discussion}

This paper discusses an assessment of the temporal geoid accuracy that is obtained from the GRACE satellite mission. For the assessment, we constructed a GRACE simulation data set where the contribution to the long- and short-periodic geoid RMS caused by air pressure errors, ocean tide errors, continental hydrology and ocean bottom pressure variations is simulated by models.

This paper is essentially a follow-up to the closed-loop procedure introduced by Visser and Schrama (2004). New in this paper is that we have chosen a combined approach where GEODYN is used to generate a 1year GRACE simulation data set. New compared to the approach of Visser and Schrama (2004) is that separate "residual orbit" and KBR post-processing techniques are used to estimate monthly geoids up to spherical harmonic degree and order 40 or 50 , depending on the simulation case.

Input to the DMI procedure, introduced in Sect.2.2, are observed GPS positions relative to an a priori dynamic orbit. We demonstrated a linear correction model that relies on FFTs and spectral tapering techniques to compute a perturbating acceleration series for frequencies up to $4 \mathrm{cpr}$ with a linear damping to 0 at $8 \mathrm{cpr}$. This perturbation spectrum is computed offline from the POD procedure. In subsequent iterations, we inserted 
DMI spectra in the POD procedure and we were able to demonstrate that we can find dynamically consistent trajectories that fit to within $1-2 \mathrm{~cm}$ to externally provided GPS kinematic and reduced dynamic solutions of CHAMP. In the last iteration of the DMI procedure, we find, typically, corrections on the level of $10 \mathrm{~nm} / \mathrm{s}^{2}$, which match orbit displacements of $3.3 \mathrm{~mm}$ radially. The conclusion is that we can always find dynamic orbits that fit to be within the GPS noise level of the input orbit.

Although we are able to recover a hydrology signal from the radial accelerations as observed from orbit residuals in the simulation set, it must be concluded that the obtained DMI acceleration spectra are too noisy for temporal gravity research since the largest science signal (hydrology) does cause radial accelerations at the $10 \mathrm{~nm} / \mathrm{s}^{2}$ level, which corresponds to $3.3 \mathrm{~mm}$ radially. For comparison, similar research on a 2-year long CHAMP data set with the "acceleration approach" (Ditmar et al. 2006) yields geoid commission errors of about $10 \mathrm{~mm}$ at degree and order 20 when their solution is compared to the more accurate EIGEN-GRACE01S solution (Reigber et al. 2002).

In order to assess the monthly geoid accuracy for GRACE, we used observation equations based upon the conservation of total residual energy approach that was introduced by Ray et al. (2003). New in our approach is a detrending correction of the perturbation potential and the projection of the velocity vector at both GRACE satellites, which involves information from the GPS residual analysis. This correction is efficient in removing a significant part of the striping effect in the monthly geoid maps and the annual amplitude and residual RMS geoid maps that are obtained by post-processing.

The main conclusion from the closed-loop simulation is that the annual hydrology can be recovered from the simulated GRACE data set and that this signal clearly stands out above the other simulated error sources; all known hydrologic dipoles are present in the recovered annual geoid. Striping patterns in the residual geoid maps are significantly reduced by (1) the use of an exact solver, (2) a priori weighting by suitable observation and parameter sigmas, and (3) the implementation of a detrending procedure prior to the estimation of the monthly geoid solutions.

Another simulation run is concerned with the recovery of OBP variations for which we have used the ECCO ocean model. In contrast to the simulation of tide and atmospheric pressure signals, OBP is generally considered to be a signal of scientific interest, i.e., a signal that we want to be able to retrieve from the GRACE data. The simulated annual OBP effect turns out to be on the $1 \mathrm{~mm}$ level locally. However, we want to mention that Chambers et al. (2004) were able to recover a global ocean mass variation effect that comes with an amplitude of about $8 \mathrm{~mm}$ for equivalent water height. From this, we conclude that a locally averaged OBP signal is affected too much by noise in the monthly GRACE geoid solutions, but the OBP signal itself is large enough to be recovered by global integration.

The simulation case based upon the ECMWF minus NCEP model differences shows that surface air pressure errors do map into a geoid effect with an RMS up to $1.5 \mathrm{~mm}$. Another remarkable, but perhaps unrealistic, feature are the larger geographic anomalies over the Antarctic. Apparently both meteorological models differ significantly in these regions. We suggest that spaceborne GPS atmospheric occultation profiling may yield unique information on the vertical structure of the refractive index and therefore the air pressure signal over the polar regions.

GRACE is not designed to sample ocean tides so that there are few possibilities to remove this type of error from the data (Ray et al. 2003). The conclusion from the FES99 minus GOT99.2 ocean tide model difference in the closed-loop simulation is that this type of error results (compared to other error sources) in patterns with an annual amplitudes of $0.5 \mathrm{~mm}$ maximally. A significant part of the non-annual tidal error is concentrated at the mid and higher latitudes. The long- and short-periodic distribution of the investigated effects displayed in the figures of Sect. 3 is summarized in Table 1.

We conclude from the long-periodic geoid RMS that continental hydrology is a large effect, and that it stands out above OBP, air pressure and tidal effects. In general, it will be difficult to separate local OBP effects from simulated air pressure and tide errors because of the relative difference in size shown in Table 1 . For periods shorter than 3 months, we find that OBP variations are larger than hydrology and simulated air pressure errors, and that tidal effects are smaller.

Table 1 The contribution of different cases in our GRACE closed-loop simulation

\begin{tabular}{lllc}
\hline Modeled effect & LP $(\mathrm{mm})$ & SP $(\mathrm{mm})$ & L/S \\
\hline Hydrology & 4.5 & 0.25 & 10 \\
Ocean bottom pressure & 0.8 & 0.4 & 2 \\
Delta air pressure & 0.5 & 0.3 & 1.5 \\
Delta tide model & 0.5 & 0.1 & 5 \\
\hline
\end{tabular}

Column LP contains the geoid RMS (in $\mathrm{mm}$ ) for periods longer than 3 months, column SP is for periods shorter than 3 months, and column L/S indicates the ratio of both effects. High L/S ratios suggest that the modeled effect is mostly long periodic, while smaller ratios indicate the opposite 
Acknowledgements Kinematic GPS orbits for CHAMP were provided by D. Švehla at the Technical University of Munich, Germany, reduced dynamic orbits for CHAMP originate from J. van den IJssel at the Technical University of Delft. The Center of Space Research at the University of Texas in Austin kindly provided the GGM01S solution, and the Space Geodesy Branch at the NASA Goddard Space Flight Center in Greenbelt, MD, the GEODYN orbit determination software. NCEP and ECMWF reanalysis products were provided by National Centers for Environmental Prediction and the European Center for Medium-Range Weather Forecasts, the ECCO ocean model was provided by the Jet Propulsion Laboratory, and Jürgen Kusche at the Technical University of Delft is acknowledged for the pre-processing of the ECCO ocean bottom pressure grids.

\section{References}

Chambers DP, Wahr J, Nerem RS (2004) Preliminary observations of global ocean mass variations with GRACE. Geophys Res Lett 31:L13310. DOI 10.1029/2004GL020461

Dickey JO, Bentley CR, Bilham R, Carton JA, Eanes RJ, Herring TA, Kaula WM, Lagerleof GSE, Rojstaczer S, Smith WHF, van den Dool HM, Wahr JM, Zuber MT (1997) Satellite Gravity and the Geosphere, National Research Council, Washington DC

Ditmar P, Kuznetsov A, van de Eck van der Sluis AA, Schrama EJO, Klees R (2006) DEOS_CHAMP_01C_70: A model of the Earth's gravity field computed from accelerations of the CHAMP satellite. J Geod 79:586-601. DOI 10.1007/s00190005-0008-6

Fan Y, van den Dool H (2004) Climate Prediction Center global monthly soil moisture data set at $0.5^{\circ}$ resolution for 1948 to present. J Geophys Res 109:D10102. DOI 10.1029/2003JD004345

Gerlach C, Sneeuw N, Visser PNAM, Svehla D (2003) CHAMP gravity field recovery with the energy balance approach: first results. In: Reigber C, Lühr $\mathrm{H}$, Schwintzer $\mathrm{P}$ (eds) First CHAMP mission results for gravity, magnetic and atmospheric studies. Springer, Berlin Heidelberg New York, pp 134-139

Han S-C, Jekeli C, Shum CK (2004) Time-variable aliasing effects of ocean tides, atmosphere, and continental water mass on monthly mean GRACE gravity field. J Geophys Res 109(B4). DOI 10.1029/2003JB002501, pp. B04403, 1-10

Han S, Shum CK, Matsumoto K (2005) GRACE observations of M2 and S2 ocean tides underneath the Filchner-Ronne and Larsen ice shelves, Antarctica. Geophys Res Lett 32:L20311. DOI 10.1029/2005GL024296

Heiskanen WA, Moritz H (1967) Physical Geodesy. Freeman, San Francisco

van den IJssel J, Visser PNAM (2003) CHAMP precise orbit determination using GPS data. Adv Space Res 31(8):1889-1895

Jekeli C (1999) The determination of gravitational potential differences from satellite-to-satellite tracking. Celest Mech Dyn Astron 75(2):85-101. DOI 10.1023/A:1008313405488

Kroes R, Montenbruck O, Bertiger W, Visser PNAM (2005) Precise GRACE baseline determination using GPS. GPS Solutions 9(1):21-31 DOI: 10.1007/s10291-004-0123-5

Lefèvre F, Lyard FH, Le Provost C, Schrama EJO (2002) FES99: a global tide Finite Element Solution assimilating tide gauge and altimetric information. J Atmos Ocean Technol 19(9): 1345-1356

Mathers EL, Woodworth PL (2001) Departures from the local inverse barometer model observed in altimeter and tide gauge data and in a global barotropic numerical model. J Geophys Res 106(C4):6957-6972
McCarthy D, Petit G (2003) IERS Conventions 2003, IERS Technical Note, Report No. 32, BundesAmt für Kartographie und Geodäsie, Frankfurt am Main. http://www.iers.org. Last accessed 21 March 2006

Montenbruck O, Gill E (2000) Satellite orbits - models methods applications. Springer, Berlin Heidelberg New York. ISBN 3540-67280-X

Pavlis DE, Moore D, Luo S, McCarthy JJ, Luthcke SB (1999) GEODYN operations manual, 5 vols. Raytheon ITSS, Greenbelt

Press WH, Flannery BP, Teukolsky SA, Vetterling WT (1989) Numerical recipes: the art of scientific computing, Fortran version. Cambridge University Press, Cambridge

Ray RD (1999) A global ocean tide model from TOPEX/POSEIDON altimetry: GOT99.2. NASA Goddard Space Flight Center, NASA/TM 209478, US government printing office

Ray RD, Rowlands DD, Egbert GD (2003) Tide models in the era of satellite gravimetry. Space Sci Rev 108:271-282

Reigber Ch, Balmino G, Schwintzer P, Biancale R, Bode A, Lemoine J-M, Koenig R, Loyer S, Neumayer H, Marty J-C, Barthelmes F, Perosanz F, Zhu SY (2002) A high quality global gravity field model from CHAMP GPS tracking data and Accelerometry (EIGEN-1S). Geophys Res Lett 29(14):1-4

Reigber Ch, Lühr H, Schwintzer P, Wickert J (2005a) Earth observation with CHAMP. Results from three years in orbit. Springer, Berlin Heidelberg New York. ISBN 3-540-22804-7

Reigber CH, Schmidt R, Flechtner F, Konig R, Meyer U, Neumayer KH, Schwintzer P, Zhu SY (2005b) An Earth gravity field model complete to degree and order 150 from GRACE EIGEN-GRACE02S. J Geodyn 39(1):1-10

Rowlands DD, Luthcke SB, Klosko SM, Lemoine FGR, Chinn DS, McCarthy JJ, Cox CM, Anderson OB (2005) Resolving mass flux at high spatial and temporal resolution using GRACE intersatellite measurements. Geophys Res Lett 32:L04310. DOI 10.1029/2004GL021908

Švehla D (2005) Kinematic positioning of LEO and GPS satellites and IGS stations on the ground. Adv Space Res. DOI 10.1016/j.asr.2005.04.066

Švehla D, Rothacher M (2005) Kinematic precise orbit determination for gravity field determination. In: Sansò F (ed) A window on the future of geodesy. Springer, Berlin Heidelberg New York, pp 181-188

Schrama EJO (1992) Some remarks of several definitions of geographically correlated orbit errors: consequences for satellite altimetry. manuscr geod 17:282-294

Schrama EJO (2003) Error characteristics estimated from CHAMP, GRACE and GOCE derived geoids and from satellite altimetry derived mean dynamic topography. Space Sci Rev 108:179-193

Seeber G (1993) Satellite geodesy: foundations, methods and applications. Walter de Gruyter, Berlin. ISBN 3-11-012753-9

Stammer D Davis R, Fu LL, Fukumori I, Giering R, Lee T, Marotzke J, Marshall J, Menemenlis D, Niiler P, Wunsch C, Zlotnicki V (1999) The Consortium for Estimating the Circulation and Climate of the Ocean (ECCO), Science Goals and Task Plan. The ECCO report series, Report No. 1, November 1999. http://www.ecco-group.org/. Last accessed 21 March 2006

Swenson S, Wahr J (2002) Methods for inferring regional surfacemass anomalies from Gravity Recovery and Climate Experiment (GRACE) measurements of time-variable gravity. J Geophys Res 107(B9):2193. DOI 10.1029/2001JB000576

Tapley BD, Bettadpur S, Ries JC, Thompson PF, Watkins MM (2004a) GRACE measurements of mass variability in the Earth system. Science 305(5683). DOI 10.1126/science. 1099192, pp. 503-505 
Tapley BD, Bettadpur S, Watkins M, Reigber Ch (2004b) The gravity recovery and climate experiment: mission overview and early results. Geophys Res Lett 31:L09607. DOI 10.1029/2004GL019920

Tapley BD, Ries J, Bettadpur S, Chambers D, Cheng M, Condi F, Gunter B, Kang Z, Nagel P, Pastor R, Pekker T, Poole S, Wang F (2005) GGM02-an improved Earth gravity field model from GRACE. J Geod 79(8):467-478. DOI 10.1007/s00190005-0480-Z

Thompson PF, Bettadpur SV, Tapley BD (2004) Impact of short period, non-tidal, temporal mass variability on GRACE gravity estimates. Geophys Res Lett 31:L06619. DOI 10.1029/2003GL019285
Velicogna I, Wahr J, van den Dool H (2001) Can surface pressure be used to remove atmospheric contributions from GRACE data with sufficient accuracy to recover hydrological signals? J Geophys Res 106(B8). DOI 10.1029/2001JB900228, 1641516434

Visser PNAM, Schrama EJO (2004) Space-borne gravimetry: how to decouple the difference gravity field constituents. In: Jekeli C, Bastos L, Fernandes J (eds) Gravity, geoid and space missions. Springer, Berlin Heidelberg New York, pp 6-11. ISBN 3-540-26930-4

Wessel P, Smith WHF (1996) A global, self-consistent, hierarchical, high resolution, shore-line database. J Geophys Res 101(B4):8741-8743 